\title{
LEAF AREA CALCULATING BASED ON DIGITAL IMAGE
}

\author{
Zhichen Li, Changying Ji ${ }^{*}$, Jicheng Liu \\ * Corresponding author: College of Engineering, Nanjing Agricultural University, Nanjing, \\ Jiangsu, 210031, China, Email: chyji@njau.edu.cn
}

\begin{abstract}
A new and simple method for plant leaf area measurement using image processing technique was provided. Sixty leaves with different shape and size of six plant species were selected for study. The software using for image processing and leaf area calculation was matlab6.0. Experiments were performed to test the performance of the estimating system by comparing the estimated leaf area with the grid square leaf area. The range of relative errors of estimation were $4.42-7.81 \%, 3.56-8.01 \%, 4.12-6.97 \%, 3.89-7.28 \%, 4.23-$ 8.12\% and 3.56-7.03\% for Eucommia Bark, Paulownia, Maidenhair tree, Bamboo, Cycad, Weed respectively. The results illuminate the more close to rectangle area the more accurate of the estimating leaf area. So it can be concluded that the method developed in this study obtained the sufficient accuracy for the possible non-destructive leaf area measurement.
\end{abstract}

Keywords: leaf area, image processing, grid square

\section{INTRODUCTION}

The leaf is the most important photosynthesis organ of plant, and it affects crop growth and bio-productivity. Leaf area is a valuable parameter in studies of plant nutrition, plant protection measure, plant soil-water relations, crop ecosystem etc. (Mohsenin, 1986). Accurate and rapid non-destructive leaf area measurement is important in plant studies of understanding and modeling ecosystem function. The methods for leaf area measurement include: 
1. Weighing. Copying the shape of the leaf on a piece of paper and weighing the copy. So leaf area is estimated by the following equation: $L A=W / c$ LA is the leaf area, $\mathrm{W}$ is the weight of the paper and $\mathrm{c}$ is the coefficient of the paper (weight of unit area).

2. Counting grid. Projecting leaf on a piece of grid paper and counting the number of panes. So Leaf Area is estimated by the following equation: $L A=G N \times G A$ LA is the leaf area, GN is the number of grid and GA is area of one grid.

3. Regression equation. This method includes two steps. Firstly one specific species of plant leaf of different size is selected for study. The length and width of the leaf is measured and the leaf area is also measured using method 1 or 2 . the leaf area prediction model was developed as the following: $\mathrm{LA}=\mathrm{f}(\mathrm{L}, \mathrm{W}) \mathrm{LA}$ is the leaf area, $\mathrm{L}$ is leaf length and $\mathrm{W}$ is leaf width. Second, when you want to know the specific plant leaf area, you can calculate the leaf area using the equation only by measuring the length and width of the leaf.

4. Photoelectric scanning. Scanning the reference object e.g. $50 \times 50 \mathrm{~mm}$ by specific dot per inch (dpi). Calculating the area of one pixel. Then scanning the leaf by the same dpi. And calculating the leaf area by the following equation: $L A=P N \times R A P \mathrm{LA}$ is the leaf area, $\mathrm{PN}$ is the number of leaf scanning image pixels and RAP is area of one reference object scanning image pixel.

5. Utilizing leaf area instrument. It is reliable and convenient to estimate leaf area using mechanical, digital or portable scanning planimeters (Daughtry, 1990).

Every method of the above is reliable, but method 1 and method 2 take much time; method 3 requires many different equations and one equation is only applicable for one specific species of plant; method 5 is expensive and method 1-5 are destructive.

Due to the developments of computer, PC-based image processing has become a feasible tool in plant studies. Digital image processing has been used for leaf area measurement. Meyer and Davidson (1987) used the stereoscopic system and measured the leaf area from the three-dimensional coordinates of edge points identified by the user-interactive program. Nyakwende et al. (1997) determined the leaf area by the regression of the project leaf areas in three viewpoints, from the top, from the side and from the oblique angle. In a series of previous research activities, a nondestructive image processing technique to measure time-dependent growth curves of selected vegetable seedlings has been developed (Chien and Lin, 2000a, 2000b, 2002). 
The leaf area measurement method introduced in this paper is rapid and simple, the metrical result is reliable. The system is not expensive and it is possible to realize non-destructive measurement.

\section{MATERIALS AND METHOD}

\subsection{Material}

The image acquisition system is depicted in figure 1. A hole was drilled in the centre of the top face of the box and the camera was placed on the hole. The diameter of the hole is a little bigger of the lens of the camera. The distance between top and bottom face is $450 \mathrm{~mm}$. It is easy for adjusting the focus of the camera because the axis of the lens is vertical to the flat. The left, right, front and back face of the box is hollow, so it is easy for the light projecting in and it is difficult for forming shadow. It is also easy for the leaf to be placed under the camera. The source of the light is the natural light. A piece of white paper with a rectangle was placed on the platbed and the side of the rectangle is black. A lot of paper with varied rectangle was prepared for the leaf of different size. The leaf on the rectangle was covered a transparent glass plate to obtain a high-contrast image of the leaf. So it is easy for getting reliable threshold for segmentation. Another function of the glass is to flat the leaf. The nut is used to tighten the glass.

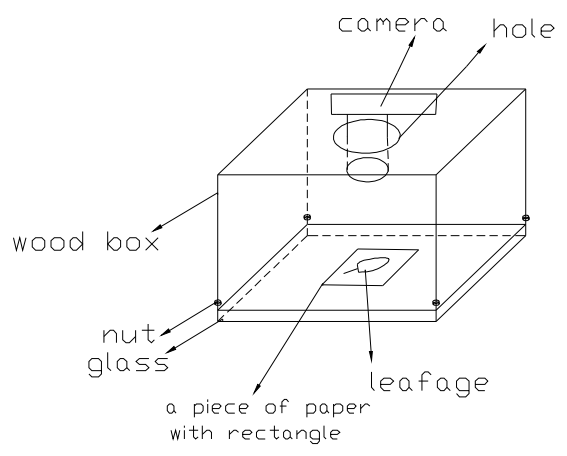

Fig. 1. Image acquisition system structure

The model of the CCD camera is Olympus N438. The image was acquired outdoors and was digitized into 24-bit (RGB) images with a resolution $640 * 480$. The image is processed by computer (Pentium III $700 \mathrm{MHz}$ CPU). The images were recorded on a U-disc of the camera and then downloaded into the personal computer. An image processing and determining leaf area program was developed using Matlab 6.0 under the Microsoft Windows operating system. 
To compare the estimated leaf area with the actual leaf area, total 60 leaves of 6 species were sampled for image acquisition and measurement. The actual leaf areas were determined by method 2 (counting grid). Leaves selected for area measurement were Cycad, Paulownia, Bamboo, Weed, Maidebhair tree, and Eucommia Bark representing varied shapes (Fig. 2). Cycad leaves are heart-shaped and Paulownia leaves are star shaped. Bamboo leaves are fish-shaped. Weed leaves are long like a sword. Maidebhair tree leaves are fan shaped and Eucommia Bark leaves are elliptical.

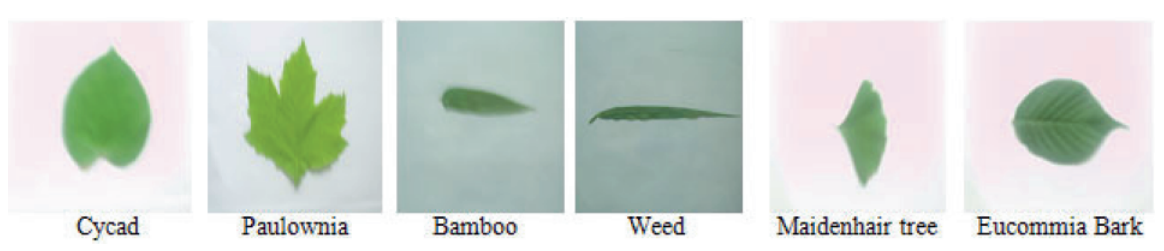

Fig. 2. Leaf image of plants

\subsection{Algorithms and Image processing}

The software algorithm for the system was written in Matlab6.0. the algorithm execute the operations of image processing including setting thresholds and binary image, the estimated leaf is segmented from surroundings by set threshold, the pixel value is set to 1 and 0 by the threshold; of pixels number counting and leaf area calculating. It is easy and rapid for counting the pixel number because the pixel value has only two, 1 and 0 .

The leaf area is estimated by the following equation.

$$
I L A=R A \times L P N / R P N
$$

ILA is leaf area based on image processing and RA is rectangle area. LPN is leaf pixel number and RPN is rectangle pixel number. Leaf area results were compared with measurements from graphical grid methods. Graph sheets with $1 \mathrm{~mm} 2$ grids were used in graphical grid methods. So the total leaf area is estimated by the following equation

$$
G L A=I S N+P S N / 2
$$

GLA is the estimated leaf area by grid method, ISN is the number of enclosed squares by the profile and PSN is the number of passed through squares by the profile.The error rate is determined by the following equation: ER is the error rate, GLA is the estimated leaf area by grid method and ILA is leaf area based on image processing.

$$
E R=((G L A-I L A) / G L A) \times 100 \%
$$




\section{RESULTS AND DISCUSSIONS}

Ten leaves of every species of plants were selected for leaf area estimation. Five leaves are normal and the other five leaves are abnormal, some leaves are incomplete and some leaves are blurred, they are shown in fig. 3. One leaf area was calculated three times. The leaf area value equals the average of the three values. The estimated leaf area was compared with the results of grid square method.

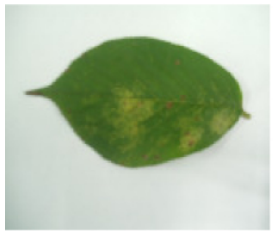

Blurred leaf

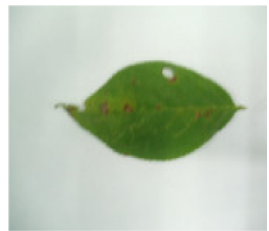

Incomplete leaf

Fig. 3. Abnormal leaf

The analyses of relative errors are included in table 1 . So it can be concluded that the result is reliable. It has the maximum error rate for the abnormal leaf area calculation and minimum error rate for the normal leaf area. The result is showed in table 2. The error rate of the abnormal leaf area is bigger than the normal leaf area. The cause is that the pixels of the blurred or incomplete leaf were set zero in the process of binary, the number of the LPN is little and then the error is big.

Table 1. The error rate of estimated leaf area

\begin{tabular}{lcccccc}
\hline Species & Eucommia bark & Paulownia & Maidenhair tree & Bamboo & Cycad & Weed \\
\hline Minimum Error rate (\%) & 4.42 & 3.56 & 4.12 & 3.89 & 4.23 & 3.56 \\
Maximum Error rate (\%) & 7.81 & 8.01 & 6.97 & 7.28 & 8.12 & 7.03 \\
Average error $(\%)$ & 5.23 & 5.02 & 4.83 & 4.96 & 5.35 & 4.92 \\
\hline
\end{tabular}

Table 2. The error rate of normal and abnormal estimated leaf area

\begin{tabular}{lcccc}
\hline \multirow{2}{*}{ Species } & \multicolumn{2}{c}{ Normal Leaf } & \multicolumn{2}{c}{ Abnormal Leaf } \\
\cline { 2 - 5 } & MinER & MaxER & MinER & MaxER \\
\hline Eucommia bark & 4.42 & 4.48 & 4.62 & 7.81 \\
Paulownia & 3.56 & 4.12 & $4 . .89$ & 8.01 \\
Maidenhair tree & 4.12 & 4.97 & 4.38 & 6.97 \\
Bamboo & 3.89 & 6.28 & 7.22 & 7.28 \\
Cycad & 4.23 & 5.68 & 4.23 & 8.12 \\
Weed & 3.56 & 7.03 & 4.56 & 7.03 \\
\hline
\end{tabular}

MinER is minimum error rate and MaxER is maximum error rate (\%)

The influence of rectangle area is also studied. Five pieces of paper with different rectangle were used for the background and reference object of the image. Three leaves for every species of plant were selected for studying. The relative error equals the average of the estimated three leaves area error value. The relations between error and rectangle area were showed in table 3 
Table 3. The relation of relative error and rectangle area

\begin{tabular}{llllll}
\hline Rectangle Area $\left(\mathrm{mm}^{2}\right)$ & 12000 & 15000 & 18000 & 21000 & 24000 \\
\hline Relative Error $(\%)$ & 4.36 & 5.36 & 6.08 & 6.25 & 6.48 \\
\hline
\end{tabular}

\section{CONCLUSIONS}

This study has shown that the accuracy of estimated leaf area is correlated to the area of the rectangle. As well as the rectangle area is a little bigger than the leaf area, a very high accurate result can be acquired. This study has also shown that the accuracy lies on the leaf characteristic, for example: normal leaf or abnormal leaf (blurred or incomplete). A high accurate result can be getting for the normal leaf area estimating.

The system we made for estimating area of plants leaf having different shapes is reliable and simple. Experiments were performed to test the performance of the estimating system by comparing the image estimated leaf area with grid square method. The method developed in this study obtained very high accuracy.

Because the study of understanding plant growth and development has been increasing, this system will be very useful tool for estimating leaf area for plants without using additive expensive devices. It is not expensive and it can utilize the existing computer and camera, therefore it does not require additional hardware. The device is very convenient for taking to field. So it is possible to enable researchers to make non-destructive and repeated measurement.

\section{REFERENCES}

Chien C.F., and T.T. Lin. 2000a. Non-destructive measurement of vegetable seedling leaf area using elliptical Hough transform. ASAE Parpe No. 003023. St. Joseph, Mich: ASAE.

Chien C.F., and T.T. Lin. 2000b. An image processing method to measure overlapped leaf area using elliptical Hough transform. J. Agric. Machinery 9 (4):47-64 (in Chinese), English summary.

Cirak C., Odabas M.S., Ayan A.K., 2004. Leaf area prediction model for summer snowflake (Leucojum aestivum) International Journal of botany.

Daughtry. 1990. Direct measurements of canopy structure. Remote Sensing reviews. 5, 45-60

Eschenbach, C., Kappen, L., Leaf area index determination in an alder forest: a comparison of three methods. J. Exp. Bot. 47, 1457-1462.

Gamiei. Y.S., Randle W.M., Milisha, Smittleda, 1991. A rapid and non-destructive method for estimating leaf area of onions. Horticultural Science, 26: 206.

Gholz, H.L., Ewel, K., Tesky, R.O., 1990. Water and forest productivity. For. Ecol. Manage. $30,1-18$.

Iakahiro Kanuma, K. Ganno, S. Hayashi etc. Leaf Area Measurement Using Stereo vision. Artificial Intelligence in Agricultural. 1998. 
Lieih J.H., James F.R., Hugo H.R., 1986. Estimation of leaf area of soybeans grown under elevated carbon dioxide levels. Field Crop Research, 13: 193-203.

Meyer G.E., and D.A., Davidson, 1987. An electronic image plant growth measurement system. Trans. of the ASAE, 30(1), 242-248.

Mohsenin N.N., 1986. Physical Properties of Plant and Animal Materials. Gordon and Breach Science Publisher. New York, pp. 107-110.

Nyakwende E.C., J. Paul and J.G. Atherton, 1997, Non-destructive determination of leaf area in tomato plants using image processing. Journal of horticultural science, 72 (2), 255-262. 